\title{
Der Erwerb der Fachkunde im Strahlenschutz und deren Erhalt
}

\section{Einführung}

Der vorliegende Beitrag setzt sich mit den Anforderungen der Fachkunde im Strahlenschutz nach der Röntgenverordnung (RöV) sowie der Strahlenschutzverordnung (StrlSchV) auseinander. Trotz oftmals langjähriger Tätigkeit und Abrechnungspraxis der Ärzte begegnet man immer wieder dem Umstand, dass diese nicht die erforderlichen Voraussetzungen erfüllen, um ihre Tätigkeit im Bereich der Röntgendiagnostik oder Röntgentherapie ausführen und rechtmäßig abzurechnen zu dürfen. Die fehlenden Fachkundevoraussetzungen bleiben den betroffenen Ärzten in der Regel unerkannt, sodass ihre Verwunderung umso größer ist, wenn die Strahlenschutzbehörde das Fehlen der Fachkundebescheinigung moniert, mit Sanktionen belegt und die Kassenärztliche Vereinigung ihrerseits die Fehlerhaftigkeit der Abrechnungspraxis rügt und fälschlich gezahltes Honorar zurückfordert. Aufgrund der für die Röntgenpraxis herausragenden Bedeutung der Fachkunde sollen die nachfolgenden Ausführungen daher die für die Fachkunde im Strahlenschutz notwendigen Voraussetzungen aufzeigen, den Weg zum Erwerb der Fachkundebezeichnung beschreiben sowie deren Erhalt schildern. Darüber hinaus soll der Beitrag eine Hilfestellung dahingehend leisten, wie im Fall der versäumten Aktualisierung oder gänzlich fehlenden Fachkundebezeichnung vorgegangen werden kann.

\section{Erwerb der Fachkunde im Strahlenschutz}

Um Leistungen in dem Bereich der Röntgendiagnostik und der Röntgentherapie erbringen und abrechnen zu dürfen, muss der Arzt einen Fachkundenachweis im Strahlenschutz erwerben. Die Fachkundebescheinigung wird ihm seitens der zuständigen Ärztekammer ausgestellt, sofern er die erforderlichen Voraussetzungen belegen und entsprechende Bescheinigungen vorlegen kann. Die nachzuweisende Fachkunde im
Strahlenschutz ist durch eine Teilnahme in eigens anerkannten Kursen ${ }^{1}$ sowie der Ableistung einer Sachkundezeit, die eine Anzahl von Mindestuntersuchungszahlen beinhaltet, zu erwerben.

Der Umfang der für den Strahlenschutz erforderlichen Fachkunde ist von der Art der beabsichtigten Tätigkeit des Arztes abhängig. Die Fachkunde im Strahlenschutz in der Röntgendiagnostik richtet sich nach $\S 18$ a Röntgenverordnung (RöV), die insbesondere den Umgang mit Röntgeneinrichtungen und Störstrahlern regelt; der Erwerb der Fachkunde in der Strahlentherapie und Nuklearmedizin richtet sich nach $\S 30$ Strahlenschutzverordnung (StrISchV), die den Umgang mit radioaktiven Stoffen und ionisierender Strahlung bestimmt. Dabei gelten für den Erwerb beider Fachkunden ähnliche Regeln, sodass die jeweiligen Grundkurse einander weitestgehend entsprechen. Gleichwohl müssen die Grundkurse für deren Anrechnung als Nachweis zum Erwerb der speziellen Fachkunde durch die Ärztekammer zwingend, entweder nach der Röntgenverordnung oder der Strahlenschutzverordnung, als solche anerkannt sein. Hierauf ist im Vorfeld bei der Auswahl der Kurse besonders zu achten. Der theoretische Teil des Kenntniskurses kann in dem Grundkurs bereits mitenthalten sein.

Die jeweiligen Spezialkurse unterscheiden sich voneinander und sind von den Ärzten in Abhängigkeit von der angestrebten Tätigkeit zu belegen. So müssen Ärzte, die etwa radiologische Untersuchungen durchführen wollen, beispielsweise Spezialkurse in der Computertomografie und Interventionsradiologie belegen. Nuklearmediziner, die künftig die Radiosynoviorthese durchführen wollen, müssen einen speziellen Kurs im Strahlenschutz zum Umgang mit offenen radioaktiven Stoffen belegen, Strahlentherapeuten einen Spezialkurs im

1 Vgl. zur Genehmigung eines Grundkurses im Strahlenschutz unter Einsatz von e-Learning VG Köln, Urt. v. 13.12.2016, Az.: 7 K 5410/14.

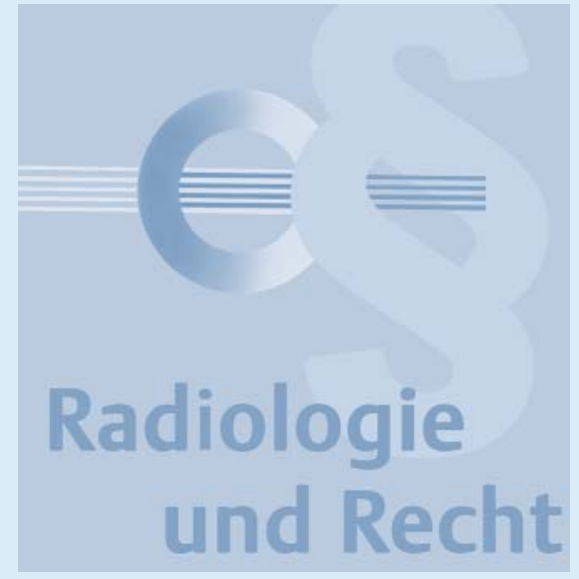

Strahlenschutz in der Brachytherapie oder, je nach Anwendungsbereich, einen Spezialkurs im Strahlenschutz in der Teletherapie oder in der Tele-, Brachy- und Röntgentherapie. An den Spezialkurs schließt die Sachkundezeit an, die sich ebenfalls unterschiedlich ausgestaltet. Für die Fachkunde in der Röntgendiagnostik umfasst sie praktische Berufserfahrung bei der Anwendung ionisierender Strahlen am Menschen und erfordert das Erlernen der rechtfertigenden Indikation, sowie der technischen Durchführung und der Befundung von Röntgenaufnahmen.

\section{Antragstellung}

Die letzte Voraussetzung für den Erwerb der Fachkunde ist die Antragstellung. Hierauf weisen die Kursleiter in der Regel in den Kursen hin, was aber nicht bedeutet, dass die fehlende Antragstellung einen Ausnahmefall darstellt. Nicht selten vergessen Radiologen die Beantragung der als Nachweis der Fachkunde dienenden Fachkundebescheinigung bei der zuständigen Ärztekammer. Diese ist für den Erwerb der Fachkunde jedoch unerlässlich und zählt ebenso zu ihren zwingenden Voraussetzungen, wie die Teilnahme an den Kursen und das Sachkundezeugnis selbst. In aller Regel handelt es sich hierbei zwar um eine reine Formalie, mitunter ist bei der Ärztekammer jedoch ein zusätzliches Fachkundegespräch erforderlich. 


\section{Aktualisierungspflicht}

Die Ärztekammer prüft das Vorliegen der Voraussetzungen zur Erteilung der Fachkundebescheinigung bei der Antragstellung, wobei die Kursteilnahme nicht länger als 5 Jahre zurückliegen darf ( $\$ 18 a$ Abs. 1 S. 4 RöV bzw. §30 Abs. 1 S. 4 StrlSchV). Darüber hinaus müssen Radiologen die Fachkunde alle 5 Jahre durch eine erfolgreiche Teilnahme an einem von der zuständigen Stelle anerkannten Kurs oder anderen von der zuständigen Stelle als geeignet anerkannten Fortbildungsmaßnahmen aktualisieren. Die Aktualisierung der Fachkunde im Strahlenschutz richtet sich dabei nach $\S 18$ a Abs. 2 RöV respektive $\S 30$ Abs. 2 StrISchV. Für die Berechnung der Aktualisierungsfrist ist als Bezugstermin das Ausstelldatum der ersten Fachkundebescheinigung zugrunde zu legen. Für die zweite und jede weitere Aktualisierung ist der letzte Kursbesuch maßgeblich. Die Frist von 5 Jahren ist tagesgenau zu berechnen. Schon ein Tag der Fristversäumnis führt dazu, dass die Frist überschritten und die Fortführung der bisherigen radiologischen Tätigkeit rechtlich nicht mehr zulässig wäre. Dies wiederum berechtigt die zuständige Stelle, die Fachkunde zu entziehen oder die Fortgeltung der Fachkunde mit Auflagen zu versehen. Der Nachweis über die durchgeführten Fortbildungen ist der zuständigen Stelle auf Anforderung vorzulegen. Wird der Nachweis über Fortbildungsmaßnahmen nicht oder nicht vollständig vorgelegt, kann die zuständige Stelle ebenfalls die Fachkunde entziehen ${ }^{2}$ oder die Fortgeltung mit Auflagen versehen. Welche Auflagen dies sind, entscheidet die Ärztekammer im Einzelfall. Im Rahmen ihrer Ermessenausübung hat sie angesichts des mit dem Entzug der Fachkundebescheinigung verbundenen Eingriffs in die Berufsausübungsfreiheit (Art. 12 Abs. 1 GG) zu berücksichtigen, ob mildere, aber gleich effektive Mittel zur Erreichung des Zwecks, d. h. die Verhinderung drohender erheblicher Gesundheitsgefahren für Leib und Leben von Pa-

2 Vgl. zur Entziehung der Fachkundebescheidung wegen fehlendem Fortbildungsnachweis OVG Nordrhein-Westfalen, Beschl. v. 5.4.2016, Az.: 13 A 2483/15 sowie mangels hinreichender Fachkunde OVG NordrheinWestfalen, Urt. v. 24.11.2016, Az.: 13 A 293 15, GesR 2017, 128. tienten infolge des nicht sachgemäßen Umgangs mit Röntgenstrahlen, in Betracht kommen.

Versäumt ein Radiologe die Aktualisierung der Kurse oder ist die Teilnahme an dem vorgesehenen Kurs fristwahrend nicht mehr möglich, verbleibt ihm die Möglichkeit, die Fachkunde „auf andere geeignete Weise" oder durch eine erfolgreiche Teilnahme an „anderen von der zuständigen Stelle als geeignet anerkannten Fortbildungsmaßnahmen“ zu aktualisieren ( $§ 18 a$ Abs. 2 S. 2 RöV und $\S 30$ Abs. 2 S. 2 StrlSchV). Gemeint ist damit die Belegung eines anderen Spezialkurses im Strahlenschutz, um die Fachkunde zu erhalten. Besucht der Radiologe einen solchen weiteren Spezialkurs und schließt diesen erfolgreich $a b$, ist künftig das Datum der Kursbescheinigung des Kurses als neuer Bezugstermin für die Frist zur Aktualisierung zugrunde zu legen. Aufgrund eines höheren Stundenumfangs des Kurses ist dies für die Betroffenen allerdings mit einem zusätzlichen zeitlichen Aufwand verbunden. Es ist daher ratsam, zunächst das Gespräch mit der Ärztekammer zu suchen und zu erfragen, ob diese den Erhalt der bestehenden Fachkunde nicht an die Pflicht zur Teilnahme am terminlich nächstgelegenen Aktualisierungskurs knüpfen kann.

\section{Entbehrlichkeit einer eigenen Fachkunde}

Einer eigenen Fachkunde bedarf es hingegen nicht, wenn sich der Arzt einem Strahlenschutzbeauftragten unterstellt und nur unter Aufsicht und Verantwortung des fachkundigen Radiologen tätig wird. In diesem Fall genügen eine praktische Einweisung durch einen Arzt mit der erforderlichen Fachkunde, über die dieser ein Zeugnis ausstellt, und ein weiterer Nachweis über die Teilnahme an einem Kenntniskurs. Dies entspricht den Voraussetzungen des Strahlenschutzes, erfüllt jedoch nicht die Voraussetzungen zur Abrechnung im Bereich der Röntgendiagnostik und der Strahlentherapie oder Nuklearmedizin als eigene Leistung gegenüber einer Kassenärztlichen Vereinigung. Die Kassenärztlichen Vereinigung bzw. die Qualitätssicherungsvereinbarung Strahlendiagnostik und -therapie verlangt vielmehr den Nachweis einer eigenen Fachkunde. Der Strahlenschutzbeauftragte dürfte die Leistung auch nicht als eigene Leistung abrechnen, da dieser den tätigen Arzt zwar beaufsichtigt, er selbst aber keine eigene abrechenbare Leistung erbringt.

\section{Fehlende \\ Fachkundebescheinigung}

Es stellt sich abschließend die Frage, wie zu verfahren ist, wenn die erforderliche Fachkundebescheinigung fehlt. In Betracht kommt ein alternativer Nachweis mittels Approbationsurkunde, sofern sie den Fachkundeerwerb durch einen Zusatz auf der Urkunde dokumentiert. Jedoch muss der Arzt auch in diesem Fall seiner fünfjährigen Aktualisierungsverpflichtung nachgekommen sein.

Liegen die Voraussetzungen für eine Fachkundebescheinigung de facto nicht vor, ist in der Regel ein Herantreten an die Ärztekammer und die Kassenärztliche Vereinigung unumgänglich. Liegen bereits erbrachte Kursteilnahmen zumindest teilweise vor, wird die Ärztekammer bestenfalls die bereits vorliegenden Nachweise anerkennen und nur vereinzelt den Neuerwerb weiterer, aktueller Kursbescheinigungen verlangen. Möglich wäre demnach, dass die Ärztekammer den Grundkurs anerkennt und nur den Neuerwerb der Bescheinigungen für die jeweiligen Spezialkurse fordert. Ein Rechtsanspruch des betreffenden Arztes auf diese Vorgehensweise besteht selbstredend nicht. Fraglich bleibt, ob die Ärztekammer sodann auch den Nachweis über die Sachkunde anerkennt oder eine erneute Ableistung verlangt. Dies wäre im Einzelfall zu prüfen. Hat ein Radiologe lediglich die Antragstellung übersehen, wäre, bei Vorlage entsprechender Aktualisierungsbescheinigungen, die Erteilung der Fachkundebescheinigung ohne Belegung weiterer Kurse zumindest denkbar. Diese hätte jedoch keine Rückwirkungsfiktion und würde nicht für den Zeitraum vor ihrer Beantragung, sondern erst ab Ausstellung der Berechtigung für die Zukunft gelten. Welche Nachweise die Kammer schließlich anerkennt und welche neu erworben werden müssen, liegt letztlich in ihrem Ermessen und ist von dem jeweiligen Einzelfall abhängig. Insoweit ist es ratsam, möglichst früh 
das Gespräch mit der Ärztekammer zu suchen.

Gleiches gilt für die Kassenärztliche Vereinigung. Diese nimmt grundsätzlich eine eigene Prüfung der Voraussetzungen für die Erteilung einer Abrechnungsgenehmigung vor und erteilt diese, wenn der Radiologe die Voraussetzungen der fachlichen Befähigung erfüllt. Hierfür untersucht die Qualitätssicherungsabteilung der Kassenärztlichen Vereinigung, ob der Arzt die fachlichen Voraussetzungen anhand von Zeugnissen nachweisen kann, die belegen, dass er die benötigten Kenntnisse im Rahmen einer Facharztweiterbildung erworben hat. Für den Betrieb von radiologischen, strahlentherapeutischen und nuklearmedizinischen Einrichtungen müssen als weitere Voraussetzungen auch die Vorgaben der Röntgenverordnung und der Strahlenschutzverordnung erfüllt sein. Diese Vorgaben prüft die Kassenärztliche Vereinigung entweder selbstständig durch eine eigene Radiologische Kommission und verlangt von dem Arzt die Vorlage einer Fachkundebescheinigung oder erkundigt sich bei der Ärztekammer, ob diese eine solche Fachkundebescheinigung erteilt hat. Für den Fall einer erteilten Abrechnungsgenehmigung bei fehlender Fachkundebescheini- gung wäre zu prüfen, ob hinsichtlich bereits abgerechneter Leistungen, nicht zuletzt unter Berücksichtigung des Amtsermittlungsgrundsatzes, gegenüber der Kassenärztlichen Vereinigung einen gewisser Vertrauensschutz des Radiologen besteht. Jedenfalls dürfte mit Bekanntwerden der fehlenden Fachkunde dieser Vertrauensschutz für die Zukunft entfallen. Eine von dem Zeitpunkt an vorsätzlich falsche Abrechnung wäre sodann strafbewährt. Ob die Kassenärztliche Vereinigung in diesem Fall rückwirkend eine Nachberechnung veranlassen könnte, lässt sich nicht abschließend beurteilen, da nicht abzuschätzen ist, inwieweit die Kassenärztliche Vereinigung den von ihr geschaffenen Vertrauensschutz anerkennt und selbst wenn sie einen solchen anerkennt, ob sie von einer Nachberechnung absieht.

\section{Fazit}

Erwirbt der Radiologe eine entsprechende Fachkunde, muss er diese durch die Teilnahme an Aktualisierungskursen regelmäBig alle 5 Jahre auffrischen. Hierbei gilt die Aktualisierungsfrist als Stichtag. Alternativ kann der Radiologe fristwahrend auch andere Spezialkurse zum Erhalt der Fachkun- de belegen. Fehlt die für die Tätigkeit und Abrechnung erforderliche Fachkunde, muss sich der Arzt an die Ärztekammer bezüglich der Erteilung der Bescheinigung wenden, die dem Arzt einzelfallbezogen mitteilt, welche Nachweise angerecht werden können und welche Kurse neu erbracht werden müssen. Hinsichtlich der Abrechnungsgenehmigung muss sich der Arzt an die Kassenärztliche Vereinigung wenden, die ihrerseits das Vorliegen der Abrechnungsvoraussetzungen prüft. Eine wissentlich unberechtigte Abrechnung, aufgrund einer bewusst fehlenden Fachkunde, ist strafbewährt.

Prof. Dr. Peter Wigge

Rechtsanwalt

Fachanwalt für Medizinrecht

Dr. Nikolaus R. Marek

Rechtsanwalt

Rechtsanwälte Wigge

Scharnhorststraße 40

48151 Münster

$++49 / 251 / 535950$

++ 49/251/5359599

kanzlei@ra-wigge.de

www.ra-wigge.de 